\title{
STATE-OF-THE-ART APPROACHES \\ TO CREATING METAL-BASED DRUGS
}

\author{
E. Milaeva \\ Department of Chemistry, Lomonosov Moscow State University, \\ 119991, Russia, Moscow, Leninsky Gory, 1-3.
}

DOI: 10.19163/MedChemRussia2021-2021-112

E-mail:milaeva@med.chem.msu.ru

The presentation will focus on novel approaches to constructing physiologically active metal-based drugs candidates based on target-oriented design, new synthetic approaches and extensive biological screenings. This study is focused on the design of hybrid compounds possessing 2,6-dialkylphenol group with dual modes of action - prooxidative and antioxidative activities. The presence of metal atom allows extensive modification including coordination to the targeted specific groups to control and tune toxicity-activity profiles. The synthesis and biological activity will be discussed. The anti/prooxidant activity has been studied in in vitro, ex vivo, in vivo experiments. We used general strategy of medicinal chemistry in the design of metal (Au, Sn) containing drug candidates, that is, rational selection of validated target, computer assisted molecular design, directed synthesis and screening.

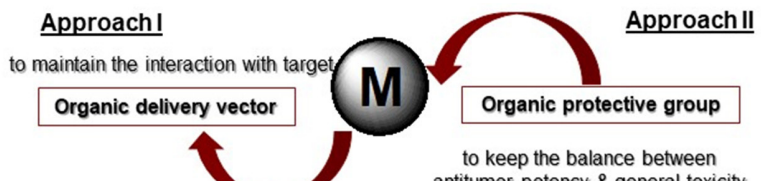
modification of the known organic drug moiety as a delivery vector by introducing a metal atom for better targeting and efficacy

\begin{abstract}
modification of the known metal compound with proved pharmacological efficacy by introducing the protective groups for attenuation of general toxicity
\end{abstract}

antitumor potency \& general toxicity

The work has generated the libraries of organic ligands and organometallic and coordination compounds. Newly synthesized compounds were studied for their physico-chemical properties, reaction capability, interaction with cellular targets including molecular docking, and finally, potency in vitro and in vivo. As a result, several lead compounds mostly organotins have been selected for further in-depth investigation.

This work was supported by RSF (19-13-00084).

\section{References}

E. R. Milaeva \& co-authors.J. Organomet. Chem., 2020, 909, 121089. Pure \& Appl. Chem., 2020, 92, 1201-1216. Res.J. Pharm. Biol. Chem. Sci., 2018, 10, 324-331. Appl. Organomet. Chem., 2018, 32, e4381. Metallomics: integrated biometal science. 2018, 10, 406-413. Eur. J. Inorg. Chem. 2017, 12, 1785-1791.Tyurin. Pure \& Appl. Chem., 2017, 89, 1065-1088. Inorg.Chim.Acta, 2017, 455, 276. Polyhedron, 2017, 127, 512. J. Organomet. Chem., 2015, 782, 96. Dalton Trans., 2015, 44, 3614. Dalton Trans., 2014, 43, 6880. Dalton Trans., 2013, 42, 19, 6817. Med. Chem. Res., 2012, 21, 3523. Dalton Trans., 2012, 41, 14568. Curr. Topics Med. Chem., 2011, 11, 2703.

$$
-112-
$$

